\title{
Calidad de Vida Relacionada con la Salud y Factores Psicológicos: Un Estudio desde la Enfermedad Pulmonar Obstructiva Crónica - EPOC
}

\author{
Health-Related Quality of Life and Psychological Factors: A Study from Chronic \\ Obstructive Pulmonary Disease - COPD
}

\author{
Stefano Vinaccia \\ Universidad Católica de Colombia, Colombia \\ $\mathrm{y}$ \\ Japcy M. Quiceno \\ Universidad de San Buenaventura Bogotá, Colombia \\ (Recibido: 21 de junio de 2010; Acep: 18 de marzo de 2011)
}

\begin{abstract}
Resumen
El objetivo de este estudio fue evaluar las relaciones entre resiliencia, percepción de enfermedad, creencias y afrontamiento espiritual-religioso sobre la calidad de vida relacionada con la salud en 40 pacientes con diagnóstico de enfermedad pulmonar obstructiva crónica de la ciudad de Medellín, Colombia. Instrumentos: escala de resiliencia RS, escala de resiliencia breve CD-RISC2, cuestionario breve de percepción de enfermedad IPQ-B, inventario de sistema de creencias SBI-15R, escala estrategias de afrontamiento espirituales SCS y cuestionario de salud MOS SF-36. A nivel de resultados hubo correlaciones positivas entre resiliencia y salud mental, y en el análisis de regresión lineal el control personal y las consecuencias de percepción de enfermedad tuvieron un peso significativo sobre el índice sumario mental de calidad de vida. En conclusión, la salud mental en crisis agudas se ve alterada por la preocupación en las consecuencias físicas, sociales, económicas y emocionales que acarrea la enfermedad, y el control personal y la resiliencia son variables que promueven la regulación emocional en pacientes con EPOC.
\end{abstract}

Palabras clave: Resiliencia, percepción de enfermedad, espiritualidad, calidad de vida, EPOC.

\begin{abstract}
The purpose of this research was to evaluate the relationship of the resilience, illness perception, beliefs and spiritual-religious coping on health-related quality of life in 40 patients with chronic obstructive pulmonary disease diagnosis from the city of Medellín, Colombia. Instruments: resilience scale RS, the connor-davidson resilience Scale CD-RISC2, the brief illness perception questionnaire IPQ-B, systems of beliefs inventory SBI-15R, spiritual coping scale SCS, medical outcomes study short form 36 ítems MOS SF-36. To level results there were positive correlations between resilience and mental health and multiple linear regression analysis personal control and consequences of illness perception had significant weight on the summary mental index of the quality of life. In conclusion, the mental health in sharp crises meets altered by the worry in the physical, social, economic and emotional consequences that it brings the disease and personal control and resilience are variables that promote the emotional regulation in patients with COPD.
\end{abstract}

Key words: resilience, illness perception, spirituality, quality of life, COPD.

Correspondencia: Stefano Vinaccia Alpi, Facultad de Psicología, Universidad Católica de Colombia, Colombia, Av. Caracas No. 46-40, Bogotá; 327 73 00. E-mail: vinalpi47@ hotmail.com.

Agradecimientos: Esta investigación se lleva a cabo gracias a la colaboración del Dr. Alberto Pérez Moreno, Coordinador del Centro de Investigaciones del Hospital General de Medellín, Luz Castro de Gutiérrez (HGM). 


\section{Introducción}

La enfermedad pulmonar obstructiva crónica (EPOC) es un término utilizado para describir dos enfermedades pulmonares relacionadas: la bronquitis crónica y el enfisema pulmonar. La bronquitis crónica se caracteriza por la inflamación prolongada del interior de los bronquios (los conductos de las vías respiratorias) y el enfisema es el ensanchamiento y destrucción irreversible de los alvéolos pulmonares (sacos aéreos). Es posible que algunas personas con EPOC manifiesten ambas enfermedades (Barnes, 2000; Parmet, 2003).

Los síntomas de la EPOC son por lo general tos crónica, aumento de la expectoración (la mucosidad que se expulsa de las vías respiratorias por medio de la tos), la disnea de esfuerzo, entre otros (Parmet, 2003). Durante el curso de la EPOC pueden aparecer otros signos y síntomas inespecíficos, como las sibilancias o la opresión torácica, la intensidad puede variar entre diferentes días, e incluso el mismo día. Estos síntomas pueden aparecer en cualquier estadio de la EPOC y acarrean como consecuencia limitaciones en la actividad física (De Calvo y Martín, 2002).

La EPOC es un problema de salud pública de enormes proporciones que está aumentando en todo el mundo por su morbi-mortalidad y costos sociales y económicos. En los últimos 30 años la mortalidad mundial por EPOC ha aumentado 163\%. La Organización Mundial de la Salud calcula que para el año 2020 la EPOC pasará de ser la causa número 12 de enfermedad en el mundo a la quinta causa de años de vida perdidos, ajustados por invalidez, y del puesto sexto como causa más común de muerte a la tercera causa de mortalidad, sólo superada por el cáncer y las enfermedades cardiovasculares, situándose incluso por delante del SIDA (INFOEPOC, 2007). Su impacto será mayor en los países en vía de desarrollo. La EPOC afecta aproximadamente a 600 millones de personas en el mundo de acuerdo con la OMS, a pesar de que la mayoría no han sido diagnosticados (Amigo, Erazo, Oyarzún, Bello y Peruga, 2006; Rennard \& Farner, 2002).

Los conocimientos sobre la prevalencia de la EPOC suelen ser pocos e incompletos en Colombia, aunque se le considera como la séptima causa de muerte en el país que va en continuo aumento, pasó del 12.8 x 100.000 habitantes en 1979 a 15.9 x 100.000 habitantes en 1994 (Maldonado, Bolívar, Caballero, Casas, Roa y Salgado, 1997). Y entre las enfermedades crónicas de vías aéreas inferiores ocupa el cuarto puesto entre las diez causas de mortalidad agrupadas según la Clasificación Internacional de Enfermedades (CIE-10) en 2001 (Durán y Vargas, 2007). En un estudio desarrollado en cinco ciudades colombianas (Bogotá, Cali, Barranquilla, Bucaramanga y Medellín) por Caballero, Torres, Maldonado, Jaramillo y Guevara (2003) se encontró que la prevalencia de la EPOC es de $8.9 \%$, considerándose como elevada. Con respecto al género esta enfermedad se presentó significativamente en hombres mayores de 60 años y en relación a la posición geográfica la prevalencia de EPOC en la ciudad de Medellín fue la más alta del país con un $13.5 \%$. En síntesis, en Colombia la EPOC es una enfermedad que afecta a la población adulta o de edad avanzada y tiene una prevalencia mayor en población urbana de sexo masculino. Es la causa mas frecuente de consulta en las personas mayores de 60 años.

La EPOC por lo común es más severa en los alcohólicos y los pacientes con enfermedad coronaria, igualmente es más probable que los pacientes con EPOC tengan antecedentes familiares de enfermedades torácicas (Álvarez et al., 2001). Además, el cigarrillo es el factor etiológico y de riesgo más importante para su desarrollo. Aproximadamente un $80-90 \%$ de casos de EPOC se atribuyen al hábito tabáquico, y un fumador tiene diez veces más probabilidades de fallecer de esta enfermedad que una persona no fumadora (Parmet, 2003). Se estima que alrededor de 30\% de la población mayor de 15 años consume cigarrillos en el mundo y en América Latina esta prevalencia es de 26\% (Amigo et al., 2006). Igualmente la contaminación ambiental originada por polvos y químicos inorgánicos y orgánicos se ha relacionado con el desarrollo de esta enfermedad como el humo (Ramírez y León, 2004).

Por lo general el diagnóstico de EPOC se realiza cuando está en un estadio avanzado (Durán y Vargas, 2007). Además de supresión del hábito de fumar, el tratamiento de la EPOC consiste en la utilización de broncodilatadores que son medicamentos administrados con frecuencia a través de un inhalador que contribuyen a abrir (dilatar) los bronquios; los glucocorticoides que son medicamentos administrados a través de un inhalador que contrarrestan la inflamación en las vías respiratorias y los pulmones; el oxígeno que ayuda a quienes tienen dificultades para obtenerlo de forma natural y suficiente; y por último, los antibióticos los cuales sirven para combatir las infecciones bacterianas que agravan los síntomas de la enfermedad (Parmet, 2003).

En cuanto a los efectos de los factores psicosociales, son diferentes en cada paciente, las consecuencias se dan con el paso del tiempo, alterando el día a día de la vida de una persona e influyendo en su visión de la vida en general. Algunas de las posibles consecuencias psicosociales encontradas en diferentes estudios son: estrés y ansiedad, depresión y bajo estado de ánimo, desesperanza, temor de morir/quedar sin aliento, pánico, alteración de la imagen corporal, cambio en la posición social dentro de la familia (donde fueron el principal sostén de la familia), el vivir solo, pérdida de control y de independencia, cambios de estilo de vida, alteración de las relaciones interpersonales, incapacidad laboral, baja autoestima/sentido de incompetencia, negación, ira, pérdida de la dignidad, frustración, culpa, pérdida de la intimidad, irritabilidad e impaciencia, vergüenza -a partir de la disnea, la tos y la producción de esputo (Kelly y Lynes, 2008). 
La expresión de la emoción está íntimamente relacionada con la disnea y algunos pacientes pueden evitarla como un mecanismo de adaptación, dando lugar al desarrollo de síntomas de ansiedad y depresión. El deterioro de la calidad de vida en las dimensiones físicas y sociales de la vida diaria resulta a menudo asociado con un estilo de vida sedentario con disnea progresiva y fatiga. Esto a su vez conduce al aislamiento social y la incapacidad de participar en muchas actividades de la vida diaria. Estos cambios son parte de una transición gradual a la dependencia e inevitablemente alteran la dinámica familiar, potenciando el desarrollo del resentimiento y el estrés (Kelly y Lynes, 2008; Maurer et al., 2008; Medinas, Más y Renom, 2009; Wilson, 2006).

La investigación ha encontrado que tanto los efectos psicosociales y fisiopatológicos de la EPOC alteran de manera significativa la calidad de vida de quienes la padecen (Breslin, 1998; Buchi, Brandli, Klingler, Klaghofer \& Buddeberg, 2000; De Miguel, 2004; Ramírez, 2007; Van Manen, Bindels, Dekker, IJzermans, Van Der Zee \& Schadé, 2002), incluso es peor que la de pacientes con diagnóstico de cáncer (Gore, Brophya, Greenstonea, 2000).

En consideración a lo anterior el propósito de este estudio fue evaluar las relaciones entre resiliencia, percepción de enfermedad, creencias y afrontamiento espiritual-religioso sobre la calidad de vida relacionada con la salud de pacientes con diagnóstico de enfermedad pulmonar obstructiva crónica de la ciudad de Medellín, Colombia.

\section{Método}

\section{Participantes}

Participaron en este estudio 40 personas de ambos géneros con diagnóstico de enfermedad pulmonar obstructiva crónica según los criterios de Dewar y Curry (2006) escogidos mediante muestreo no aleatorio de sujetos disponibles hospitalizados en el Hospital General de Medellín, Luz Castro de Gutiérrez.

Diseño: Ex post facto transversal correlacional tipo encuesta

\section{Procedimiento}

Previo permiso y autorización de los diferentes representantes científicos, se lleva a cabo la investigación en la unidad donde se encontraban hospitalizados los pacientes con EPOC. No se consideraron pacientes con deterioro cognitivo. Los pacientes que aceptaron participar voluntariamente se les comunicó sobre los objetivos de la investigación para la obtención de la firma del consentimiento informado. Las pruebas psicotécnicas fueron aplicadas de manera individual con la ayuda del investigador a cargo. La duración aproximada de aplicación de los instrumentos fue de una hora y treinta minutos.

\section{Instrumentos}

Cuestionario de Percepción de Enfermedad -IPQ-B: desarrollado originalmente por Broadbent, Petrie, Main \& Weinman (2006) y traducido al idioma español por Guic (2007). Este cuestionario esta formado por 9 ítems con un sistema de respuesta tipo likert que va de 0 a 10 puntos. Comprende 8 dimensiones como: consecuencias, duración, control personal, control del tratamiento, identidad, preocupación, respuesta emocional y comprensión de la enfermedad. Y tiene además una pregunta abierta ordinal (ítem 9) que hace relación a los factores que considera la persona que causaron su enfermedad.

Escala de Resiliencia -RS: desarrollado inicialmente por Wagnild y Young (1993) en Estados Unidos. La versión en idioma español fue desarrollada por Heilemann, Lee y Kury (2003). La escala consta de 25 ítems con un sistema de respuesta tipo likert que va de 1 a 7 puntos y comprende dos dimensiones: competencia personal (17 ítems) y aceptación de sí mismo y de la vida (8 ítems) y una escala total de los 25 ítems. A mayor puntuación mayores niveles de resiliencia.

Escala de Resiliencia Breve-CD-RISC2: desarrollada originalmente por Vaishnavi, Connor y Davidson (2007) derivada de la escala de resiliencia CD-RISC de Connor y Davidson (2003) de la cual se cuenta con una versión en idioma español (Menezes de Lucena, Fernández, Hernández, Ramos y Contador, 2006). La escala consta de 2 ítems y una sola dimensión con un sistema de respuesta tipo likert que va de 0 ("nada de acuerdo") a 4 ("totalmente de acuerdo"). A mayor puntuación mayores niveles de resiliencia.

Inventario de Sistema de Creencias -SBI-15R: este inventario fue desarrollado originalmente por Holland et al., (1998) en Estados Unidos. La versión en español fue realizada en México por Almanza, Monroy, Bimbela, Payne y Holland (2000). Comprende 15 ítems con un sistema de respuesta tipo likert que va de 0 a 3 puntos. El inventario esta compuesto por dos factores: el factor I mide creencias y prácticas religiosas y espirituales (CPRE) con 10 reactivos, el rango total es de 0 a 30 puntos; y el factor II mide el apoyo social religioso, derivado de la comunidad que comparte esas creencias (SSR) con 5 reactivos, el rango total es de 0 a 15 puntos. Las puntuaciones globales del inventario comprenden un rango de 0 a 45 puntos. A mayor puntuación mejor convicción de las creencias y prácticas religiosas y espirituales y soporte social religioso.

Escala de Estrategias de Afrontamiento Espirituales -SCS: esta escala fue desarrollada en Inglaterra por Baldacchino y Buhagiar (2003). Consta de 20 ítems con un sistema de respuesta likert que va de 0 (nunca), 1 (algunas veces), 2 (a veces) a 3 (a menudo). Comprende dos factores: Factor I mide estrategias de afrontamiento religioso $(9$ ítems), y el factor II mide estrategias de afrontamiento no 
religioso (11 ítems). A mayor puntuación un mejor afrontamiento espiritual religioso o no religioso.

Cuestionario de Salud MOS SF-36: los autores originales de este cuestionario son Ware y Sherbourne (1992) del Health Institute New England Medical Center de Boston Massachussets. Los autores de la versión 1 en español son Alonso, Prieto y Antó (1995) y Alonso, Regidor, Barrio, Prieto, Rodríguez y De la Fuente (1998). Este cuestionario comprende 36 ítems con sistema de respuesta tipo likert que van de 1 a 5 . Esta conformado por 8 dimensiones: 1. Función física; 2. Rol físico; 3. Dolor corporal; 4. Salud general; 5. Vitalidad; 6. Función social; 7. Rol emocional; y 8. Salud mental. Por otro lado, el MOS SF-36 comprende dos Î́ndices Sumarios: el índice de salud físico y el índice mental. Mayor puntuación en las dimensiones e índices sumarios del cuestionario indica mayor calidad de vida.

\section{Resultados}

Para el análisis de datos de las diferentes variables que comprendió la investigación se empleó el paquete estadístico SPSS versión 15.0. A continuación se presentan los análisis descriptivos del estudio de las variables sociodemográficas, clínicas y psicológicas como son la media, mediana, desviación típica (D.T) y las puntuaciones mínimas y máximas de cada uno de los instrumentos psicométricos como el respectivo alfa de Cronbach. Y respecto a las variables categóricas, se le sacaron porcentajes. En su orden se presenta además los resultados de correlación de Pearson y del análisis de regresión lineal múltiple.

\section{Descripción de la muestra según las características sociodemográficas y clínicas}

Se presentan en la Tabla 1 los datos descriptivos de las variables sociodemográficas y clínicas. Se puede apreciar en cuanto al género una ligera prevalencia del femenino sobre el masculino con una edad media de 69 años, un nivel de estudios de básica primaria incompleta y de estado civil casado $(42,5 \%)$. Con respecto a la variable ocupación se evidencia mayor agrupamiento en la categoría amas de casa $(52,5 \%)$ quienes viven en su mayoría con la familia
(95\%). El nivel del estrato socioeconómico colombiano fue bajo $(92,5 \%)$. El tiempo de diagnóstico de la enfermedad fue de 6 años. En relación al sistema de salud la gran mayoría $(87,5 \%)$ estaban adscritos al Sistema de Selección de Beneficiarios para Programas Sociales (SISBEN). Todos recibían tratamiento farmacológico y no psiquiátrico. Y en cuanto a la pregunta ¿Por qué está usted hospitalizado? la mayoría reportó que por asfixia.

\section{Análisis descriptivo de las variables psicológicas}

Se observan en la Tabla 2 los datos descriptivos de las variables psicológicas y el alfa de Cronbach de los instrumentos empleados. Se observa que la resiliencia (RS y CDRISC2) y las creencias y afrontamiento espiritual religiosos (SCS y SBI-15R) tuvieron respecto a las puntuaciones mínimas y máximas halladas en las escalas puntuaciones altas. En cuanto a la calidad de vida (MOS SF-36) se evidencia en orden de los resultados encontrados puntuaciones bajas en: rol físico, función física, rol emocional, índice sumario físico y mental, vitalidad y dolor corporal. Y puntuaciones medias en: salud general, función social y salud mental. Siendo el rol físico la dimensión con peores puntuaciones, mientras que la función social y la salud general tuvieron los mejores resultados. Respecto a la "evaluación declarada de la salud" (ítems 2 MOS SF-36) los pacientes consideraron que su salud estaba entre mucho peor a algo pero que hace un año (ver Tabla 3). Con relación a la percepción de enfermedad (IPQ-B) las puntuaciones más desfavorables estuvieron comprendidas en la dimensión consecuencias y preocupación, la mejor percepción estuvo en la dimensión control de tratamiento. El resto de dimensiones como duración, control personal, identidad, comprensión de la enfermedad y respuesta emocional tuvieron puntuaciones moderadas. De acuerdo al ítem 9 del IPQ-B (pregunta abierta de tipo cualitativo) que hace referencia a las "causas de la enfermedad" según el paciente, se clasificaron las respuestas de la muestra del estudio en nueve categorías. Se puede apreciar en la Tabla 4 que hubo una mayor frecuencia en las categorías "estilos de vida inadecuados" seguida por "trabajo" y por factores "trascendentales". 
Pulmonar Obstructiva Crónica -EPOC

Tabla 1.

Características sociodemográficas y clínicas de los pacientes de EPOC.

\begin{tabular}{|c|c|c|c|c|c|}
\hline & $\mathrm{n}$ & $\%$ & & $\mathrm{n}$ & $\%$ \\
\hline \multicolumn{6}{|l|}{ Género } \\
\hline Femenino & 22 & 55 & & & \\
\hline Masculino & 18 & 45 & & & \\
\hline Edad (años) & & & Escolaridad (en años) & & \\
\hline Media (DE) & 68,9 & $(3,8)$ & Media (DE) & 3,8 & 2,7 \\
\hline Rango (Min-Max) & $(42-86)$ & & Rango (Min-Max) & $(1-11)$ & \\
\hline Estado civil & & & Ocupación & & \\
\hline Soltero & 8 & 20 & Empleado & 2 & 5 \\
\hline Casado & 17 & 42,5 & Trabajador independiente & 1 & 2,5 \\
\hline Separado/Divorciado & 6 & 15 & Jubilado/Pensionado & 7 & 17,5 \\
\hline Unión Libre & 1 & 2,5 & Desempleado & 9 & 22,5 \\
\hline Viudo & 8 & 20 & Ama de Casa & 21 & 52,5 \\
\hline Con quien vive & & & Estrato Socioeconómico & & \\
\hline Solo & 2 & 5 & Bajo (nivel 1 y 2) & 37 & 92,5 \\
\hline Con familia & 38 & 95 & Medio (nivel 3 y 4 ) & 3 & 7,5 \\
\hline Con amigos & 0 & 0 & Alto (nivel 5 y 6) & 0 & 0 \\
\hline \multicolumn{3}{|l|}{ Tiempo de diagnóstico (meses) } & \multicolumn{3}{|l|}{ Sistema de Salud donde está adscrito } \\
\hline Media (DE) & 71,4 & $(101,9)$ & SISBEN & 35 & 87,5 \\
\hline Rango (Min-Max) & $(1-420)$ & & EPS & 5 & 12,5 \\
\hline \multirow[t]{2}{*}{ Media (DE) } & 71,4 & $(101,9)$ & Medicina Prepagada & 0 & 0 \\
\hline & & & Particular/Privada & 0 & 0 \\
\hline Recibe tratamiento farmacológico & & & Consume medicación psiquiátrica & & \\
\hline $\mathrm{Si}$ & 40 & 100 & $\mathrm{Si}$ & 0 & 0 \\
\hline No & 0 & 0 & No & 40 & 100 \\
\hline \multicolumn{6}{|l|}{ ¿Porque está usted hospitalizado? } \\
\hline Gripe & 9 & 24,0 & & & \\
\hline Asfixia & 31 & 76,0 & & & \\
\hline
\end{tabular}

Nota $\mathrm{n}=$ número de participantes; DT = Desviación Típica; Min. = Mínimo; Max.= Máximo 
Tabla 2.

Resultados descritivos y Alfa de Cronbach de las diferentes dimensiones de los instrumentos utilizados en el estudio.

\begin{tabular}{|c|c|c|c|c|c|c|}
\hline & Media & Mediana & DT & Mín & Máx & Alfa \\
\hline \multicolumn{7}{|l|}{ Escala de Resiliencia (RS) } \\
\hline Competencia personal & 100,4 & 102,0 & 15,9 & 46 & 119 & ,939 \\
\hline Aceptación de sí mismo y la vida & 47,0 & 48,0 & 8,3 & 24 & 56 & ,900 \\
\hline RS TOTAL & 147,3 & 150,0 & 23,5 & 70 & 175 & ,959 \\
\hline Escala de Resiliencia Breve (CD-RISC2) & 6,8 & 8,0 & 1,8 & 2 & 8 & ,950 \\
\hline \multicolumn{7}{|l|}{ Escala de Estrategias de Afrontamiento Espirituales (SCS) } \\
\hline Estrategias de afrontamiento religioso & 23,6 & 25,5 & 4,9 & 3 & 27 &, 821 \\
\hline Afrontamiento No Religioso & 30,5 & 33,0 & 4.2 & 17 & 33 &, 825 \\
\hline \multicolumn{7}{|l|}{ Inventario de Sistema de Creencias (SBI-15R) } \\
\hline creencias y prácticas religiosas y espirituales (CPRE) & 29,2 & 30,0 & 3,3 & 10 & 30 & ,959 \\
\hline apoyo social religioso (SSR) & 14,0 & 15,0 & 2,5 & 5 & 15 & ,879 \\
\hline SBI-15R TOTAL & 43,1 & 45,0 & 5,3 & 15 & 45 & ,942 \\
\hline \multicolumn{7}{|l|}{ Cuestionario MOS SF-36 } \\
\hline Función física (PF) & 30,6 & 20,0 & 32,6 & 0 & 100 & ,918 \\
\hline Rol físico (RP) & 8,1 & 0,0 & 26,8 & 0 & 100 & ,978 \\
\hline Dolor corporal (BP) & 44,9 & 41,5 & 38,8 & 0 & 100 & ,955 \\
\hline Salud general $(\mathrm{GH})$ & 55,0 & 59,5 & 28,8 & 0 & 100 & ,928 \\
\hline Vitalidad (VT) & 37,5 & 20,0 & 28,1 & 10 & 100 & ,976 \\
\hline Función social (SF) & 56,3 & 50,0 & 27,6 & 25 & 100 & ,998 \\
\hline Rol emocional (RE) & 28,3 & 0,0 & 35,8 & 0 & 100 &, 763 \\
\hline Salud mental (MH) & 50,5 & 36,0 & 33,0 & 0 & 100 & ,968 \\
\hline Índice Sumario Físico (PCS) & 33,9 & 31,2 & 10,4 & 20,7 & 61 & \\
\hline Índice Sumario Mental (MCS) & 37,0 & 33,3 & 15,7 & 15 & $74,3-$ & \\
\hline \multicolumn{7}{|l|}{ Cuestionario de Percepción de Enfermedad (IPQ-B) } \\
\hline Consecuencias & 8,8 & 10,0 & 2,5 & 1 & 10 & \\
\hline Duración & 5,2 & 5,0 & 3,3 & 0 & 10 & \\
\hline Control personal & 6,3 & 7,0 & 3,1 & 0 & 10 & \\
\hline Control de tratamiento & 8,1 & 9,0 & 2,6 & 0 & 10 & \\
\hline Identidad & 6,4 & 8,0 & 3,2 & 0 & 10 & \\
\hline Preocupación & 7,0 & 8,0 & 3,4 & 0 & 10 & \\
\hline Comprensión de la enfermedad & 6,6 & 8,0 & 3,6 & 0 & 10 & \\
\hline Respuesta emocional & 6,3 & 8,0 & 3,7 & 0 & 10 & \\
\hline
\end{tabular}

Nota : DT = Desviación Típica; Mín. = Mínimo; Máx.= Máximo

Tabla 3.

Items 2 MOS SF-36: Evaluación declarada de la salud.

\begin{tabular}{lr}
\hline Evaluación declarada de la salud & $\mathrm{n}(\%)$ \\
\hline Mucho mejor ahora que hace un año & $9(22,5 \%)$ \\
Algo mejor ahora que hace un año & $4(10,0 \%)$ \\
Más o meno igual que hace un año & $7(17,5 \%)$ \\
Algo peor ahora que hace un año & $6(15,0 \%)$ \\
Mucho peor ahora que hace un año & $14(35,0 \%)$
\end{tabular}


Tabla 4.

Ítem 9 IPQ-B: Frecuencias totales de los factores que causaron la enfermedad según los pacientes de EPOC.

\begin{tabular}{|c|c|c|}
\hline Ítem No. 9 del IPQ-B & & $\mathrm{n}(\%)$ \\
\hline 1. No sabe/No responde: & Ignora las razones por las cuales se enfermó & $4(10 \%)$ \\
\hline 2. Genética/Herencia: & $\begin{array}{l}\text { Aduce que la enfermedad es biológica especialmente de su primer grado de consan- } \\
\text { guinidad (padres) y esta asociada a la edad avanzada. }\end{array}$ & $3(8 \%)$ \\
\hline 3. Enfermedad física: & $\begin{array}{l}\text { La enfermedad diagnosticada tiene comorbilidad o fue resultado de otra enfermedad, } \\
\text { por ejemplo, enfermedades virales y gripes }\end{array}$ & $6(15 \%)$ \\
\hline 4. Emociones negativas: & $\begin{array}{l}\text { Ansiedad, depresión, síntomas de tristeza, estrés, temores, rabia, ira/hostilidad y } \\
\text { sumisión. }\end{array}$ & $2(5 \%)$ \\
\hline 5. Servicio médico: & Diagnóstico y tratamientos m édicos percibidos por el paciente como inadecuados. & $0(0 \%)$ \\
\hline 6. Estilos de vida inadecuados: & $\begin{array}{l}\text { Consumo de alcohol, consumo de tabaco, mojarse acalorado o por lluvia, gripas } \\
\text { mal cuidadas, trasnocho, indisciplina, desorden en los hábitos de alimentación y } \\
\text { descuido con la salud. }\end{array}$ & $30(75 \%)$ \\
\hline 7. Eventos vitales estresantes: & $\begin{array}{l}\text { Situación económica difícil, eventos traumáticos en la infancia, fallecimiento de } \\
\text { familiares cercanos (cónyuge e hijos) y relaciones familiares conflictivas. }\end{array}$ & $4(10 \%)$ \\
\hline 8. Trabajo: & $\begin{array}{l}\text { Trabajo en túneles/minas de carbón, fundiciones y cañadulzales (hombres) y expo- } \\
\text { sición al humo de leña (mujeres). }\end{array}$ & $13(33 \%)$ \\
\hline 9.Trascendental: & Castigo divino, voluntad de Dios y el destino & $8(20 \%)$ \\
\hline
\end{tabular}

\section{Análisis de correlación de Pearson}

En la Tabla 5 se observan los análisis de correlación de Pearson donde hubo la existencia de relación estadísticamente significativa tanto a nivel de 0,05 y 0,01 entre las diferentes variables del estudio.

En cuanto a la percepción de enfermedad hubo correlaciones positivas entre consecuencias con resiliencia (CD-RISC2) y entre duración y control personal con afrontamiento no religioso. De igual modo se evidencia que control personal se relaciona positivamente con salud mental e índice sumario mental, y el control de tratamiento con salud general y función social. Mientras que hubo correlaciones negativas entre consecuencias con rol emocional e índice sumario mental; y entre identidad y respuesta emocional con dolor corporal; y entre identidad, preocupación y respuesta emocional con salud mental e índice sumario mental. También se observa que la respuesta emocional tuvo correlación negativa con rol emocional. Se evidencia además que algunas dimensiones de calidad de vida como salud general y salud mental tuvieron correlaciones positivas pero débiles con la resiliencia. También se encontró que el apoyo social religioso y el afrontamiento no religioso tuvo correlaciones positivas con salud mental de calidad de vida.

En cuanto a las dos escalas de resiliencia (CD-RISC2 y RS) utilizadas en el estudio se evidencia que tuvieron correlaciones positivas fuertes entre sus diferentes dimensiones y puntuación total. De igual modo se evidencia este mismo comportamiento con las escalas de creencias y afrontamiento espiritual-religioso (SBI-15R TOTAL y SCS) que tuvieron correlacione positivas entre sí. 
Tabla 5.

Análisis de correlación de Pearson entre las diferentes variables del estudio.

\begin{tabular}{|c|c|c|c|c|c|c|c|c|c|}
\hline & CD-RISC2 & $\mathrm{S}$ & CS & & & lidad de $\mathrm{v}$ & ida (MOS SI & -36) & \\
\hline Variables & Resiliencia & $\begin{array}{c}\text { Afrontamiento } \\
\text { religioso }\end{array}$ & $\begin{array}{l}\text { Afrontamiento } \\
\text { NO religioso }\end{array}$ & $\begin{array}{c}\text { Dolor } \\
\text { Corporal }\end{array}$ & $\begin{array}{c}\text { Salud } \\
\text { General }\end{array}$ & $\begin{array}{c}\text { Función } \\
\text { Social }\end{array}$ & $\begin{array}{c}\text { Rol } \\
\text { emocional }\end{array}$ & $\begin{array}{c}\text { Salud } \\
\text { Mental }\end{array}$ & $\begin{array}{l}\text { Índice suma- } \\
\text { rio mental }\end{array}$ \\
\hline Consecuencias &, $316^{*}$ & & & & & & ,394* & & ,357* \\
\hline Duración & & & ,336* & & & & & & \\
\hline Control personal & & &, $352 *$ & & & & &, $477 * *$ &, $414 * *$ \\
\hline Control de tratamiento & & & & &, $360 *$ &, $314^{*}$ & & & \\
\hline Identidad & & & &,$- 533 * *$ & & & &,$- 449 * *$ &,$- 370 *$ \\
\hline Preocupación & & & & & & & &,$- 441 * *$ &,$- 400 *$ \\
\hline Respuesta emocional & & & &,$- 315^{*}$ & & &,$- 356 *$ &,$- 347 *$ &,$- 355^{*}$ \\
\hline Competencia personal &, $778 * *$ & & & &, $375^{*}$ & & &, $350 *$ & \\
\hline $\begin{array}{l}\text { Aceptación de sí mis- } \\
\text { mo y de la vida }\end{array}$ &, $640 * *$ & & & & ,394* & & & & \\
\hline Resiliencia Total &, $750 * *$ & & & &, $391 *$ & & &, $334 *$ & \\
\hline CD-RISC2 & & & & & & & & ,320* & \\
\hline $\begin{array}{l}\text { Creencias y prácticas } \\
\text { religiosas y espirituales }\end{array}$ & &, $821 * *$ &, $672 * *$ & & & & & & \\
\hline Apoyo social religioso & &, $749 * *$ &, $863 * *$ & & & & &, $337 *$ & \\
\hline SBI-15R TOTAL & &, $866 * *$ &, $828 * *$ & & & & & & \\
\hline $\begin{array}{l}\text { Afrontamiento NO } \\
\text { religioso }\end{array}$ & & & & & & & & ,373* & \\
\hline
\end{tabular}

$* \mathrm{p}<0,05$

$* * \mathrm{p}<0,01$

\section{Análisis de Regresión Lineal Múltiple}

Respecto a los modelos finales de regresión lineal múltiple se encontró que el índice sumario mental presenta un coeficiente de determinación $\mathrm{R}^{2} \mathrm{c}=28 \%$; explicado positivamente por la dimensión control personal -IPQ-B $(\beta=0,439 ; \mathrm{t}=3,231 ; p=0,003)$ y negativamente por la dimensión consecuencias -IPQ-B $(\beta=-0,386 ; \mathrm{t}=-2840 ; p$ $=0,007)$. Y, en ninguno de los modelos estimados con el método de selección por pasos para el índice sumario físico del cuestionario de salud MOS SF-36, quedaron incluidas las variables del estudio, ya que no fueron estadísticamente significativas para el modelo ( $\mathrm{p}>0,05)$.

\section{Discusión}

La mayoría de los pacientes de este estudio fueron adultos mayores de 69 años en adelante, mujeres amas de casa, quienes viven con sus familias, de estado civil casado, de estrato socioeconómico colombiano bajo y con tres años de estudios solamente ( $3^{\circ}$ primaria), inscritos en el SISBEN y con un tiempo de diagnóstico de enfermedad de 6 años. Respecto a lo anterior, los datos de este estudio no difieren mucho de lo encontrado por Caballero et al., (2003), donde los factores de riesgo de EPOC en Colombia fueron: la edad mayor de 60 años, el antecedente de tuberculosis, ninguna escolaridad, el sexo masculino, a diferencia de este estudio que fue el sexo femenino, el hábito de fumar y la exposición a humo de leña por más de 10 años (como se indicará más adelante) y variaciones geográficas. Siguiendo este estudio se planteó además que en la ciudad de Medellín la prevalencia de EPOC fue la más alta en toda Colombia.

Respecto a la resiliencia (RS) hubo niveles altos en las distintas escalas. Estos resultados llevan a pensar por un lado que los pacientes del estudio tienen habilidades y capacidades personales como de auto-confianza, independencia, decisión, invencibilidad, poderío, ingenio, y por otro tienen capacidad de perseverancia, adaptabilidad, balance, flexibilidad y una perspectiva de vida estable para lograr la auto aceptación tanto de sí mismo como de la vida a pesar de la adversidad (Connor \& Davidson, 2003; Wagnild \& Young, 1993). Numerosos estudios con enfermos crónicos han hecho relevante el papel de la resiliencia como factor protector ante circunstancias difíciles de la vida (Friborg, Hjemdal, Rosenvinge, Martinussen, Aslaksen, \& Flaten, 2006; Rabkin, Remien, Katoff \& Williams, 1993; White, Driver \& Warren, 2008; Zautra, Johnson \& Davis, 2005).

Es particularmente interesante señalar que las escalas RS y CD-RISC2 han mostrado evidencias de validez de constructo convergente por la asociación significativa 
observada entre ellas. Estos resultados son particularmente importantes ya que abren un camino a la investigación de las medidas psicométricas en resiliencia, ya que aún hay poca información sobre las relaciones entre escalas de resiliencia de autores distintos (Ahern, Kiehl, Sole \& Byers, 2006; Ospina, 2007).

Respecto a las escalas de creencias y afrontamiento espiritual religioso se encontró niveles altos. Evidenciándose en estos pacientes que en tiempos de enfermedad las creencias religiosas se han fortalecido, tienen la creencia en un poder más alto, oran por su enfermedad con otros o solos y han encontrado un propósito en la vida a través del autocuidado de la enfermedad. Para estos pacientes es también muy importante el ser miembro de un grupo guía que comparta las mismas creencias y practicas religiosas/espirituales como medio de apoyo social y que brinde ayuda y aprobación o consuelo en momentos de dificultad como estrategia de afrontamiento para sobrellevar la enfermedad como se ve reflejado en las correlaciones, donde hubo relaciones positivas pero más bien débiles entre apoyo social religioso y la salud mental del MOS SF-36. El resultado de este estudio es afín a lo informado en otras investigaciones con enfermos crónicos (Harvey \& Silverman, 2007; McCauley, Tarpley, Haaz \& Bartlett, 2008). Es de anotar además que los pacientes del estudio forman parte de un mismo contexto cultural "la subcultura andina de la montaña" también llamada "cultura paisa" que basa sus principios en "los valores tradicionales de la familia, la religión como pilar central, la unidad grupal, el trabajo duro y la afiliación" (Gutiérrez de Pineda, 1999).

De igual modo que las escalas de resiliencia se evidenció en este estudio que las dos escalas religiosas/espirituales tuvieron fuerte relación (SBI-15R y SCS). Lo significativo es la relación hallada entre las creencias y practicas religiosas y espirituales y el afrontamiento no religioso. Estos resultados parecen paradójicos, sin embargo los ítems que hacen referencia al segundo factor del cuestionario SCS -afrontamiento no religioso- hacen relevancia más a aspectos trascendentales de la espiritualidad que a un afrontamiento no religioso. Al respecto según Bonanno (comunicación personal, 10 de abril, 2009) las medidas a través de cuestionarios de autoinforme de espiritualidad y afrontamiento religioso actualmente existente son a veces limitados para medir el constructo espiritualidad en toda su dimensión. Siguiendo por este análisis se podría pensar entonces que en momentos críticos de la vida -como es el caso de estos pacientes- las personas buscan respuestas más allá de sus límites humanos y empiezan a percatarse, a dar significado y valor a las cosas sencillas de la vida como apreciar la naturaleza y las artes, utilizar la reflexión para identificar las potencialidades personales, encontrar un propósito en la vida y ver el lado positivo de la situación presente para mitigar los efectos físicos y emocionales de la enfermedad. Particularmente en este estudio se evidencia que los pacientes están buscando otras estrategias más allá de sus límites personales para alcanzar un mejor control personal ante la enfermedad y también para sobrellevar la incertidumbre de no tener claro el marco temporal (duración) de su enfermedad y para tener un equilibrio en su salud mental como se evidenció a nivel del análisis correlacional. $\mathrm{Al}$ respecto, Reed (2003) argumenta que en momentos de enfermedad, de edad avanzada y frente a eventos vitales estresantes aumenta la vulnerabilidad, es decir la conciencia de mortalidad que lleva a la autotrascendencia, o sea a la ampliación de los limites autoconceptuales consigo mismo, el mundo y los demás (Coward, 2007).

Otro aspecto importante a considerar en este estudio fue la ausencia de relaciones encontradas entre resiliencia y las creencias y afrontamiento espiritual religioso. Lo que difiere con otros estudios con muestras de enfermos crónicos donde sí las han hallado (Feder, Nestler \& Charney, 2009).

En cuanto a la percepción de la enfermedad se encontró que estos pacientes estaban altamente preocupados y atentos a los signos y síntomas físicos y emocionales de la enfermedad y como estos podían alterar y modificar su funcionamiento físico, social y situación económica (consecuencias). Además se evidencia que fueron receptivos al tratamiento médico (control de tratamiento) y trataban de manejar su enfermedad en función de sus capacidades (control personal) sin tener totalmente claro la evolución y el tiempo de duración de su enfermedad (duración).

De acuerdo a las causas de la enfermedad (IPQ-B, ítem 9) los pacientes de EPOC consideraron los estilos de vida inadecuados (más concretamente el consumo de cigarrillo) y el trabajo en minería y el humo del carbón de leña como influyentes. Ahora bien, parecería que estos pacientes "comprendieran su enfermedad" por los resultados de esta dimensión en el IPQ-B, sin embargo este punto hay que analizarlo con cuidado ya que los pacientes no perciben su enfermedad como crónica sino como una infección vírica de las vías respiratorias, de corta duración y con síntomas y signos molestos etiquetados como "asfixia y gripe" como se evidencia en los datos clínicos. Es por tanto, posible, que la "causa" de la enfermedad en este caso -el cigarrillo-genere conductas de salud distintas a las esperadas frente a una enfermedad crónica. El solo creer en algo (por ejemplo: el cigarrillo es nocivo para la salud) no es suficiente para llegar a hacer una evaluación cognitiva de la experiencia que posibilite una mejor comprensión de la enfermedad. Al respecto, Howard Leventhal descubrió que el modo en el cual se representa o se comprende la amenaza de la enfermedad para vida o el temor que esta suscita resultaba a veces efectivo para producir un plan de acción (Marks, Murray, Evans, Willig, Woodall \& Sykes, 2008). Las teorías sobre la percepción de enfermedad y cognitivas argumentan además que después del análisis de la búsqueda de causas de salud y/o enfermedad enmarcadas de acuerdo a la gravedad de la misma se puede lograr el entendimiento de esta 
y la búsqueda de conductas y estrategias de afrontamiento encaminadas hacia la salud (Álvarez, 2002; Weinman \& Petrie, 1997).

En cuanto a la calidad de vida se evidencia que los sentimientos de energía y vitalidad están disminuidos por la presencia frecuente de cansancio y agotamiento que disminuyen el rendimiento esperado en todas las actividades. Sin embargo se percibe a nivel correlacional que los síntomas y signos de la enfermedad (identidad) están asociados negativamente con la salud mental, especialmente el dolor corporal y a mayor dolor y preocupación por la enfermedad más vulnerabilidad ante respuestas emocionales negativas.

A pesar de las particularidades de la enfermedad la función social, la salud mental y la salud general en estos pacientes no se ven tan alteradas como lo esperado respecto a las otras dimensiones de calidad de vida. Lo que indica posiblemente que la enfermedad no ha interferido significativamente en las relaciones interpersonales, y la salud mental está siendo regulada aunque hayan síntomas de dolor, lo que posiblemente es explicado por la visión optimista que tienen estos pacientes de su salud general actual, y de las perspectivas de la salud en el futuro y la resistencia en enfermar. $\mathrm{Al}$ respecto, diferentes estudios han planteado que las personas en la etapa adulta y en etapas posteriores es cuando alcanzan mayor bienestar y satisfacción con la vida, manejan mejor la enfermedad a nivel emocional ya que han adquirido mayor experiencia en el afrontamiento de eventos estresantes a través de los años y los problemas de salud constituyen un tipo de situación estresante habitual y, por lo tanto, esperable o predecible por la persona, por lo que la adaptación resultaría más fácil (Blanchflower \& Osvald, 2008; Cruz y Torres, 2006; Leventhal, 1984; Yanguas, 2006).

Ahora bien, se puede explicar la relación positiva hallada entre salud general y resiliencia ya que estas dos variables tienen como característica común el optimismo. Y si hay una visión optimista de la enfermedad es posible lograr la regulación en la salud mental en momentos críticos como se evidencia en los análisis de correlación. Por otro lado, se percibe a nivel de análisis correlacional que la adaptación a los cambios y la capacidad de recuperación frente a la enfermedad, es decir la resiliencia, pueden posibilitar un cierto control sobre los pensamientos catastróficos acerca de las consecuencias físicas, sociales y económicas percibidas de la enfermedad y las consecuencias emocionales sentidas. La resiliencia podría ser uno de los factores para explicar por lo tanto "la paradoja del bienestar" que ocurre cuando las personas enfermas y mayores con limitaciones en el funcionamiento cotidiano informan todavía un buen nivel de bienestar (Netuveli \& Blane, 2008). Se evidencia además que la función social juega un rol significativo para los pacientes de EPOC en los momentos de hospitalización para poder tener un mejor control en su tratamiento. A nivel general en este estudio se puede decir que la resiliencia está amortiguando los efectos negativos de la enfermedad a nivel de la salud mental de la calidad de vida lo que es afín con la literatura (Brix, Schleußner, Füller, Röhrig \& Strauß, 2009; Brix, Schleußner, Füller, Röhrig, Wendt, \& Strauß, 2008; Friborg et al., 2006; Strauss et al., 2007; Zautra et al., 2005).

De acuerdo a diversas investigaciones en pacientes con diagnóstico de EPOC donde se evaluó la calidad de vida con el cuestionario MOS SF-36 se encontró que todas las dimensiones estaban por debajo de las medias poblacionales excepto en las dimensiones rol físico y rol emocional, a diferencia de este estudio donde estas dos dimensiones tuvieron las puntuaciones medias más bajas en todo el cuestionario (Alonso et al., 1998). Es de resaltar que en este estudio la muestra fue evaluada durante una fase aguda de la enfermedad en proceso de hospitalización, y esto explica por qué perciben que su salud está "mucho peor ahora que hace un año" (ítem 2 MOS SF-36). Doll y Miravitlles (2005) en una revisión de literatura sobre estudios de CVRS en EPOC encontraron que esta enfermedad se asocia con un compromiso importante de la calidad de vida relacionada con la salud inclusive en pacientes con enfermedad leve. Por otro lado, los enfermos incluidos en todas estas investigaciones refirieron peor calidad de vida durante las exacerbaciones agudas. En uno de estos estudios, tres cuartas partes de los enfermos tuvieron una CVRS desfavorable durante las exacerbaciones agudas. Las dimensiones de función física, función emocional, salud general y vitalidad del MOS SF-36 se vieron afectadas en forma particular durante estos episodios. Por lo general, la gravedad y frecuencia de las crisis agudas aumentan en el curso de la patología, mientras se deteriora de manera constante el estado de salud general.

Con relación a los índices sumario físico y mental del MOS SF-36 se evidencia mayor sintomatología de emociones negativas y deterioro físico lo que corrobora nuevamente el ítem 2 del MOS SF-36 donde consideran que están mucho peor que hace un año. En estos pacientes es común que se presente una superposición de síntomas entre las dificultades respiratorias asociadas a la enfermedad (disnea, ahogo, sensación de asfixia, dolor toráxico) y niveles de estrés y ansiedad (Wyszynski \& Weiner, 2006). Es importante anotar en este estudio que el control personal coadyuva a una mejor salud mental mientras que la preocupación por las consecuencias físicas, sociales, económicas y emocionales que acarrea la enfermedad la deterioran como se percibe a nivel correlacional y en los análisis de regresión lineal, donde estas dos dimensiones de percepción de enfermedad tuvieron un peso significativo sobre el índice sumario mental.

Las creencias de control pueden ayudar a los procesos de adaptación y hasta puede prolongar la vida de quien padece una determinada enfermedad. En diferentes estudios con enfermos crónicos se han reportado que quienes creen tener el control sobre su enfermedad se ajustan mejor a su 
condición física y psicosocial que aquellos que carecen de esta creencia. En estudios desarrollados con pacientes de EPOC se ha encontrado que la sensación de control y autoeficacia ha sido relacionada como una forma de mejorar la adaptación (Taylor, 2007). En otros estudios se ha hallado que aquellos pacientes de EPOC con altas expectativas de autoeficacia sobrevivieron por más tiempo que aquellos sin esas expectativas, estos resultados sugieren fuertemente que cuando una enfermedad es percibida como modificable y bajo el control personal, el proceso de recuperación de las enfermedades crónicas es más favorable en el tiempo (Kohler, Fish, \& Greene, 2002). En definitiva, las creencias de control pueden llevar a individuos enfermos a poner en práctica conductas más saludables, a conseguir niveles más altos de adherencia al tratamiento y en alcanzar una mejor calidad de vida (Beneit \& Latorre, 1994; Marks et al., 2008).

En consideración a los resultados encontrados, sería interesante desarrollar estudios comparativos entre pacientes en crisis/hospitalizados y de consulta externa con las variables que comprendió este estudio para analizar las diferencias.

Los resultados de este estudio pueden servir de base para el diseño y desarrollo de futuros programas de intervención que aborden las cogniciones catastróficas sobre la enfermedad y que trabajen sobre el control personal para regular las respuestas emocionales negativas.

\section{Referencias}

Ahern, N.R., Kiehl, E.M., Sole, M.L., \& Byers, J. (2006). A review of instruments measuring resilience. Issues in Comprehensive Pediatric Nursing, 29, 103-125.

Almanza, J., Monroy, M., Bimbela, A., Payne, D.K., \& Holland, J.C. (2000). Spanish Version of the Systems of Belief Inventory (SBI15-R): Cross Cultural Research on Spiritual and Religious Beliefs. Psychosomatics, 41, 158.

Alonso, J., Prieto, L., \& Antó, J.M. (1995). La versión española del SF-36 Health Survey (Cuestionario de Salud SF-36): Un instrumento para la medida de los resultados clínicos. Medicina Clínica, 104, 771-776.

Alonso, J., Regidor, E., Barrio, G., Prieto, L., Rodríguez, C., \& De la Fuente, L. (1998). Valores poblacionales de referencia de la versión española del Cuestionario de Salud SF-36. Medicina Clínica, 111, 410-416.

Álvarez, J. (2002). Estudio de las creencias, salud y enfermedad: Análisis psicosocial. México, D.F: Trillas.

Álvarez, J.L., Cimas, E., Masa, J.F., Miravitlles, M., Molina, J., \& Naberan, K., et al., (2001). Recomendaciones para la atención al paciente con enfermedad pulmonar obstructiva crónica. Archivios de Bronconeumología, 37, 269-278.

Amigo, H., Erazo, M., Oyarzún, M., Bello, S., \& Peruga, A. (2006). Tabaquismo y enfermedad pulmonar obstructiva crónica: Determinación de fracciones atribuibles. Revista Médica de Chile, 134, 1275-1282.

Baldacchino, D.R., \& Buhagiar, A. (2003). Psychometric evaluation of the Spiritual Coping Strategies scale in English, Maltese, back-translation and bilingual versions. Journal of Advanced Nursing, 42, 558-570.

Barnes, P. (2000). Chronic Obstructive Pulmonary Disease. New England Journal Medicine, 343, 269-280.

Beneit, P.J., \& Latorre, J.M. (1994). Representación mental de la enfermedad. En J.M. Latorre \& P. J. Beneit (Eds.). Psicología de la salud (pp. 215-224). Buenos Aires, Argentina: Lumen.
Blanchflower, D.G., \& Oswald, A.J. (2008). Is Well-being U-Shaped over the Life Cycle?. Social Science \& Medicine, 66, 1733-1749.

Breslin, E. (1998). Percepción de la fatiga y la calidad de vida en pacientes con EPOC. Chest, 114, 958-964.

Brix, C., Schleußner, C., Füller, J., Röhrig, B., \& Strauß, B. (2009). Fatigue and its determinants in Radio-Oncology. Psychotherapie, Psychosomatik, Medizinische Psychologie, 59, 42-49.

Brix, C., Schleußner, C., Füller, J., Röhrig, B., Wendt, T.G., \& Strauß, B. (2008). The need for psychosocial support and its determinants in a sample of 3 patients undergoing radiooncological treatment of cáncer. Journal of Psychosomatic Research, 65, 541-548.

Broadbent, E., Petrie, K.J., Main, J., \& Weinman, J. (2006). The Brief Illness Perception Questionnaire. Journal of Psychosomatic Research 60, 631- 637.

Buchi, S., Brandli, O., Klingler, K., Klaghofer, R., \& Buddeberg, C. (2000). Inpatient rehabilitation in inpatients with chronic obstructive lung diseases (COPD): Effect on physical capacity for work, psychological wellbeing and quality of life. Schweiz Med Wochenschr, 130, 135-42.

Caballero, A.S., Torres, C., Maldonado, D., Jaramillo, C., \& Guevara, D. (2003, octubre). Prevalencia de la enfermedad pulmonar obstructiva crónica en cinco ciudades colombianas. Trabajo presentado en $\mathrm{X}$ Congreso Colombiano de Neumología y Cirugía del tórax, Ciudad de Cartagena, Colombia.

Connor, K.M., \& Davidson, J.R. (2003). Development of a new resilience scale: The connor davidson resilience scale (CD-RISC). Depression and Anxiety, 18, 76-82.

Coward, D. (2007). Teoría de la autotrascendencia. En A. Marriner \& M. Raile (Eds.). Modelos y teorías en enfermería (pp. 447-467). Madrid, España: Elsevier.

Cruz, J., \& Torres, J. (2006). “¿De qué depende la satisfacción subjetiva de los colombianos?”. Cuadernos de Economía, 45, 131-154.

De Calvo, E., \& Martin, A. (2002). Atención al paciente con asma y EPOC en la consulta del medico de familia. Barcelona, España: Masson

De Miguel, J. (2004). Calidad de vida en la enfermedad pulmonar obstructiva crónica. Archivos de Bronconeumología, 40, 480-82.

Dewar, M., \& Curry, R.W. (2006). Chronic Obstructive Pulmonary Disease: Diagnostic considerations. American Family Physician, 73, 669-678.

Doll, H., \& Miravitlles, M. (2005). Health-related QOL in acute exacerbations of chronic bronchitis and chronic obstructive pulmonary disease. A review of the literature. Pharmacoeconomics, 23, 345-363.

Durán, D., \& Vargas, O.C. (2007). La enfermedad respiratoria crónica: Reflexiones en el contexto del sistema de salud colombiano. Revista Ciencias de la Salud, 5, 106-115.

Feder, A., Nestler, E.J., \& Charney, D.S. (2009). Psychobiology and molecular genetics of resilience. Nature Reviews, 10, 446-457.

Friborg, O., Hjemdal, O., Rosenvinge, J.H., Martinussen, M., Aslaksen, P.M., \& Flaten, M.A (2006). Resilience as a moderator of pain and stress. Journal of Psychosomatic Research 61, 213-219.

Gore, J.M., Brophya, C.J., \& Greenstonea, M.A. (2000). How well do we car for patients with end-stage chronic obstructive pulmonary disease (COPD)? A comparison of palliative care and quality of life in COPD and lung cancer. Thorax, 55, 1000-1006.

Guic, E. (2007). El Cuestionario Breve de Percepción de Enfermedad. Recuperado en junio 2, 2010 de disponible en: http://www.uib.no/ipq/

Gutiérrez de Pineda, V. (1999). Estructura, función y cambio de la familia colombiana. Medellín: Universidad de Antioquia.

Harvey, I.S., \& Silverman, M. (2007). The role of spirituality in the selfmanagement of chronic illness among older african and whites. Journal of Cross Cultural Gerontology, 22, 205-220.

Heilemann, M.V., Lee, K., \& Kury, F.S. (2003). Psychometric properties of the Spanish version of the Resilience Scale. Journal of Nursing Measurement, 11, 61-72.

Holland, J.C., Kash, K.M., Passik, M.K., Gronert, M.K., Sison, A., Lederberg, M., et al., (1998). A brief spiritual beliefs inventory for use in quality of life research in Life-Threatening Illness. Psychooncology, 7, 460-469.

INFOEPOC (2007). ¿Qué es la EPOC? Datos epidemiológicos. Recuperado en mayo 3, 2007 de, disponible en: http://www.infoepoc.com/ html/queEs.htm 
Kelly, C., \& Lynes, D. (2008) Psychological effects of chronic lung disease. Nursing Times, 104: 47, 82-85.

Kohler, C.L., Fish, L., \& Greene, P.J. (2002). The relationship of perceived self-efficacy to quality of life in chronic obstructive pulmonary disease. Health Psychology, 21, 610-614.

Leventhal, E.A. (1984). Aging in the perception of illness. Research and Aging, 6, 119-135.

McCauley, J., Tarpley, M.J., Haaz, S., \& Bartlett, S.J. (2008). Daily spiritual experiences of older adults with and without arthritis and the relationship to health outcomes. Arthritis \& Rheumatism, 59, 122-128.

Maldonado, D., Bolívar, F., Caballero, A., Casas, A., Roa, J., y Salgado, C. (1997). Recomendaciones para el diagnóstico y el manejo del paciente con enfermedad pulmonar obstructiva crónica (EPOC). Revista Colombiana de Neumología, 9, 1-39.

Marks, D., Murray, M., Evans, B., Willig, C., Woodall, C., \& Sykes, C. (2008). Psicología de la salud: Teoría, investigación y práctica. México, D.F.: Manual Moderno.

Maurer, J., Rebbapragada, V., Borson, S., Goldstein, R., Kunik, M.E., \& Yohannes, A.M., et al., (2008). Anxiety and depression in COPD: current understanding, unanswered questions, and research needs. Chest, 134, 43S-56S.

Medinas, M.M., Más, C., \& Renom, F. (2009). Estrés en ancianos hospitalizados con enfermedad respiratoria crónica: Valoración en el hospital sociosanitario. Revista española de geriatría y gerontología, 44, 130-136.

Menezes de Lucena, V., Fernández, B., Hernández, L., Ramos, F., \& Contador, I. (2006). Resiliencia y modelo de burnout: Engagement en cuidadores formales de ancianos. Psicothema, 18, 791-796.

Netuveli, G., \& Blane, D. (2008). Quality of life in older ages. British Medical Bulletin, 85, 1-14.

Ospina, D.E. (2007). La medición de la resiliencia. Investigación y Educación en Enfermería, 25, 58-65.

Parmet, S. (2003). Enfermedad pulmonar obstructiva crónica. JAMA, 290, 2362.

Rabkin, J.G., Remien, R., Katoff, L., \& Williams, J.B. (1993). Resilience in adversity among long-term survivors of AIDS. Hospital Community Psychiatry, 44, 162-167.

Ramírez, R. (2007). Calidad de vida y enfermedad pulmonar obstructiva crónica. Revista Ciencia y Salud, 5, 90-100.

Ramírez, A., y León, T. (2004). Impacto del crecimiento industrial en la salud de los habitantes de una ciudad minera del Perú. Anales de la Facultad de Medicina, 65, 111-118.
Reed, P.G. (2003). The theory of Self-Transcendence. In M.J. Smith \& P. Liehr (Eds.). Middle range theories in nursing (pp. 105-130). New York: Springer.

Rennard, S., \& Farner, S. (2002). COPD in 2001. A mayor challenge for medicine, the pharmaceutical industry, and Society. CHEST, 121, 113-115.

Strauss, B., Brix, C., Fischer, S., Leppert, K., Füller, J., \& Roehrig, B., et al., (2007). The influence of resilience on fatigue in cancer patients undergoing radiation therapy (RT). Journal Cancer Research Clinical Oncology, 133, 511-518.

Taylor, S. (2007). Psicología de la Salud (6 ${ }^{\mathrm{a}}$. ed.). México, D.F.: McGrawHill.

Vaishnavi, S., Connor, K., \& Davidson, J.R.T. (2007). An abbreviated version of the connor-davidson resilience scale (CD-RISC), the CD-RISC2: Psychometric properties and applications in psychopharmacological trials. Psychiatry Research, 152, 293-297.

van Manen, J.G., Bindels, P.J., Dekker, F.W., IJzermans, C.J., Van Der Zee, J.S., \& Schadé, E. (2002). Risk of depression in patients with chronic obstructive pulmonary disease and its determinants. Thorax, 57, 412-416.

Wagnild, G.M., \& Young, H.M. (1993). Development and psychometric evaluation of the resilience scale. Journal of Nursing Measurement, $1,165-178$

Ware, J.E., \& Sherbourne, C.D. (1992). The MOS 36-ítem short form health survey (SF-36). Medical Care, 30, 473-483.

Weinman, J., \& Petrie, K.J. (1997). Illness perceptions: A new paradigm for psychosomatics? Journal of Psychosomatic Research, 42, 113-116.

White, B., Driver, S., \& Warren, A.M. (2008). Considering resilience in the rehabilitation of people with traumatic disabilities. Rehabilitation Psychology, 53, 9-17.

Wilson, I. (2006). Depression in patients with COPD. International Journal of $C O P D, 1,61-64$.

Wyszynski, A., \& Weiner, E (2006). Pacientes con neumopatías. En A. Wyszynski \& B. Wyszynski (Eds.). Manual de psiquiatría para pacientes con enfermedades médicas (pp. 89-104). Barcelona, España: Masson

Yanguas, J.J. (2006). Análisis de la calidad de vida relacionada con la salud en la vejez desde una perspectiva multidimensional. Madrid, España: Instituto de Mayores y Servicios Sociales (IMSERSO).

Zautra, A.J., Johnson, L.M., \& Davis, M.C. (2005). Positive affect as a source of resilience for women in chronic pain. Journal of Consulting and Clinical Psychology, 73, 212-220. 